\title{
Atenuação de artefatos na migração reversa no tempo (RTM)
}

\author{
Cintia Queiroz Alves ${ }^{1}$, Felipe Timóteo da Costa ${ }^{1}$, Marco Antonio Cetale Santos ${ }^{1}$ \\ ${ }^{1}$ GISIS-UFF
}

Copyright 2019, SBGf - Sociedade Brasileira de Geofísica.

This paper was prepared for presentation at the $16^{\text {th }}$ International Congress of the Brazilian Geophysical Society, held in Rio de Janeiro, Brazil, August 19-22, 2019.

Contents of this paper were reviewed by the Technical Committee of the $16^{\text {th }}$ International Congress of The Brazilian Geophysical Society and do not necessarily represent any position of the SBGf, its officers or members. Electronic reproduction or storage of any part of this paper for commercial purposes without the written consent of The Brazilian Geophysical Society is prohibited.

\section{Resumo}

A migração reversa no tempo (RTM) é um método que utiliza a extrapolação dos campos de onda completos. Dessa forma, pode-se gerar imagens de estruturas geológicas mais complexas. Apesar desse melhor imageamento, a RTM apresenta artefatos inerentes ao método. Neste trabalho, empregou-se duas abordagens para atenuar os artefatos presentes na RTM utilizando a condição de imagem por tempo de excitação. Primeiramente, foi utilizado um filtro passa banda, visando a atenuação dos ruídos de baixa frequência. $O$ segundo método abordado foi o filtro laplaciano, muito citado na literatura para atenuar os artefatos de baixa frequência ao mesmo tempo que ressalta as altas frequências. Todos os processos realizados foram aplicados na seção migrada após 0 empilhamento.

\section{Introdução}

O método sísmico de reflexão comumente utilizado na exploração de recursos minerais, consiste na emissão de pulsos sísmicos em direção a subsuperfície através de fontes sísmicas artificiais. Esses pulsos são refletidos à medida que encontram camadas geológicas com diferentes impedâncias sísmicas. As reflexões contêm informações das camadas geológicas em subsuperfície que são registradas por receptores localizados na superfície. Os dados sísmicos registrados são processados de modo a gerar imagens que representam as estruturas geológicas em subsuperfície. Entretanto as imagens formadas nesse processo sofrem diversos efeitos de distorção, em geral, as difrações devido ao truncamento das camadas geológicas e o desvio lateral dos eventos provocados pelas camadas mergulhantes. A migração é o método que corrige essas distorções, movendo as posições aparentes dos refletores na imagem sísmica para as suas verdeiras posições (YILMAZ, 2001; GRAY, 2001; CLAERBOUT, 2010).

Entre os diversos tipos de migração, a migração reversa no tempo (reverse time migration - RTM) apresenta algumas vantagens em relação aos demais métodos, como por exemplo, a sua capacidade de lidar corretamente com as variações laterais de velocidades e meios geologicamente complexos (ZHANG; SUN, 2009).
Entretanto, além do alto custo computacional envolvido (BULCÃO, 2004), a RTM apresenta artefatos inerentes a sua implementação, como por exemplo a introdução de baixas frequências e as anomalias presentes na matriz de tempo de trânsito que prejudicam a construção da imagem final. Esses artefatos ocorrem devido à presença das ondas mergulhantes e também das ondas de retroespalhamento que sofrem correlações cruzadas indesejadas na formação da imagem (GUITTON et al., 2006). Esses eventos são especialmente fortes quando tem-se um contrate alto de velocidade, que normalmente é o cenário geológico onde utiliza-se a RTM.

Portanto, neste trabalho foram aplicadas duas abordagens distintas com o objetivo de atenuar os artefatos da RTM. A primeira estratégia foi a aplicação de um filtro passa banda, visto que a RTM produz artefatos de baixa frequência. Esse filtro tem como propósito selecionar as frequências que priorizem a construção de uma imagem mais coerente. A segunda estratégia utiliza o filtro laplaciano que é recomendado em processamentos de imagens segundo diversos autores (ZHANG; SUN, 2009).

\section{Metodologia}

\section{Modelagem Sísmica}

A modelagem sísmica é um método que visa simular a propagação de ondas em meios físicos. Essa técnica é utilizada em diversos problemas geofísicos, como por exemplo: ajustes de parâmetros de aquisição, estudos de iluminação, caracterização de fraturas, estudos dos efeitos da anisotropia, entre outros. Além disso, a modelagem sísmica é utilizada como núcleo de diversas técnicas de migração sísmica (BULCÃO, 2004).

A simulação da propagação de ondas pode ser feita utilizando a equação da onda acústica. Nessa formulação apenas as ondas compressionais (ondas $\mathrm{P}$ ) se propagam no meio físico. Apesar dessa simplificação, as ondas acústicas representam com bastante precisão os fenômenos sísmicos em meios geológicos podendo ser adotada em diversos estudos (BARTOLO, 2010). A equação da onda acústica possui a seguinte expressão:

$$
\frac{1}{c^{2}(\mathbf{x})} \frac{\partial^{2}}{\partial t^{2}} p(\mathbf{x}, t)-\nabla^{2} p(\mathbf{x}, t)=f\left(\mathbf{x}_{s}, t\right),
$$

onde $\mathbf{x}$ representa as coordenadas espaciais, $t$ a variável temporal e $c(\mathbf{x})$ o modelo velocidade que contém as informações das estruturas geológicas. $p(\mathbf{x}, t)$ é o campo de pressão que se propaga no meio e $f\left(\mathbf{x}_{s}, t\right)$ é a assinatura da fonte que gera o pulso sísmico. 


\section{Migração Reversa no Tempo}

Os métodos de migração baseados na equação da onda são recomendados quando as estruturas geológicas estudadas são complexas e apresentam fortes variações laterais de velocidade, visto que os métodos baseados no traçado de raios apresentam dificuldades nestas regiões. A figura 1 apresenta os princípios básicos da migração RTM. Na aquisição sísmica, a fonte gera uma pertubação que se propaga em direção à subsuperfície (propagação direta). As reflexões que ocorrem devido às diferentes impedâncias são registradas pelos receptores na superfície, dando origem ao sismograma. Os eventos refletidos são gravados nos sismogramas, e então, propagados reversamente no tempo. Em seguida, compara-se os campos direto e reverso, utilizando uma condição de imagem e onde há uma correlação entre os campos propagados e retropropagados a imagem é formada (YILMAZ, 2001; PANIAGUA; QUINTERO; SIERRA-SOSA, 2017).

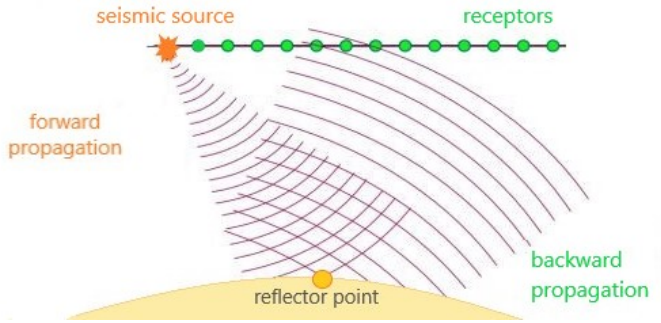

Figura 1: Esquema mostrando o princípio da RTM onde há a propagação e retropropagação dos campos de onda.

A construção de uma imagem que represente a geologia de subsuperfície depende de diversos fatores. Como por exemplo o modelo matemático utilizado para realizar a propagação do campo de ondas, e principalmente, a aplicação de uma condição de imagem adequada. Vale a pena ressaltar que, diferentemente de algumas técnicas de migração, a RTM não necessita de modelos de velocidades com grande detalhamento das estruturas geológicas existentes na área de interesse. Ela precisa de boas estimativas das velocidades presentes nesses modelos, onde as interfaces das diversas estruturas estão suavizadas (BULCÃO; Soares Filho; MANSUR, 2001). Essa característica da migração reversa no tempo a coloca entre as migrações mais utilizadas na indústria, visto que é extremamente complexa a construção de um modelo de velocidades que contenha informações altamente detalhadas das estruturas geológicas existentes em subsuperfície.

\section{Condição de Imagem}

A migração reversa no tempo utiliza em seu algoritmo sismogramas condicionados (ruídos atenuados e onda direta removida) como dados de entrada. Para evitar as diversas etapas de pré condicionamento dos dados, os dados utilizados na aplicação da migração foram gerados sinteticamente.

A condição de imagem tem a finalidade de gerar em cada ponto da malha de discretização uma imagem que ao final produzirá a seção migrada com as informações de subsuperfície. A escolha da condição de imagem é fundamental para a qualidade da imagem migrada. Entre as mais utilizadas estão: a condição de imagem por tempo de excitação e a condição de imagem com correlação cruzada (BULCÃO, 2004).

Segundo Silva (2009), a condição por tempo de excitação baseia-se no tempo que os campos de ondas atingem cada ponto da malha a partir da fonte sísmica (Figura 2). Os tempos são registrados na matriz de tempo de trânsito (Figura 3) e comparados com os tempos retropropagados dos sismogramas. A imagem de um refletor é construída quando existe a coincidência entre os tempos da matriz de tempo de trânsito e do campo retropropagado.

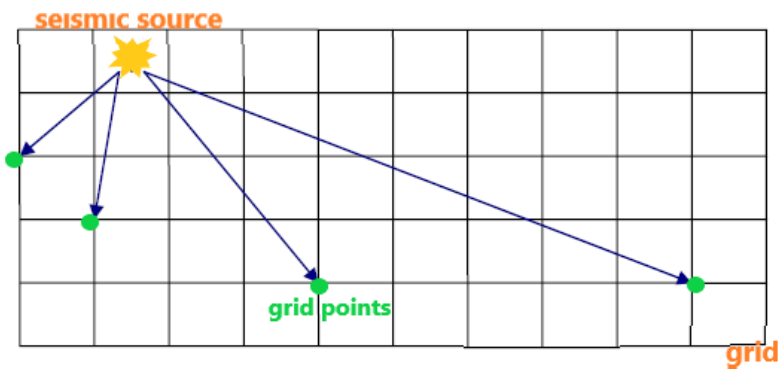

Figura 2: Tempo de chegada dos campos de ondas propagados. Modificado de Silva (2009).

A condição de imagem por tempo de excitação por ser escrita da seguinte forma:

$$
I(\mathbf{x})=\sum_{t=0}^{t=t_{f}} p_{r e v}(\mathbf{x}, t) \beta[t-T(\mathbf{x})]
$$

onde, $\mathbf{x}$ representa as variáveis espaciais, $t$ é o tempo da depropagação do campo de ondas $p_{\text {rev }}$ usando os sismogramas como fonte, $T(\mathbf{x})$ é a matriz de tempo de trânsito e $\beta$ é uma função que vale 1 se $t$ for igual a $T(\mathbf{x})$ e 0 caso contrário, por fim, $I(\mathbf{x})$ é a matriz contendo a imagem em profundidade. Neste trabalho, a matriz de tempo de trânsito foi construída através do critério de máxima amplitude.

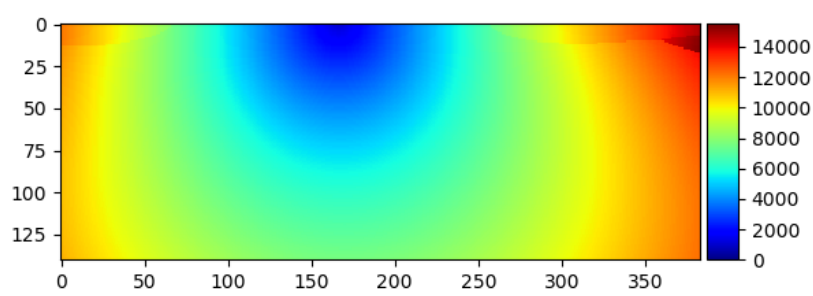

Figura 3: Matriz de tempo de transito para um modelo homogêneo.

\section{Filtro passa banda e filtro laplaciano}

Os artefatos provocados pelas baixas frequências podem ser atenuados por um filtro passa banda, que selecionam o conteúdo de frequência do dado sísmico de acordo com os parâmetros de corte selecionados. A faixa de frequência é delimitada por uma frequência de corte 
mínima $\left(f_{\min }\right)$ e uma frequência de corte máxima $\left(f_{\max }\right)$. Os valores de frequência entre esses limites do sinal sísmico são preservados, enquanto os valores de frequência fora desse intervalo são atenuados.

Outra forma muito citada na literatura para a atenuação dos artefatos é a aplicação do filtro laplaciano na imagem migrada. A vantagem de se utilizar o laplaciano é devido a sua habilidade de remover os artefatos inerentes da RTM, incluindo os de baixa frequência, enquanto preserva os mergulhos íngremes, importantes nas estruturas complexas (ZHANG; SUN, 2009). O filtro laplaciano é definido como a aplicação da derivada espacial a imagem migrada, a equação a seguir apresenta o caso 2D:

$$
I(x, y)=\nabla^{2} I(x, y)=\frac{\partial^{2} I}{\partial x^{2}}+\frac{\partial^{2} I}{\partial z^{2}}
$$

\section{Resultados}

Os testes realizados nesse trabalho utilizaram o modelo Marmousi (Figura 4a), que foi criado em 1988 pelo Instituto Francês de Petróleo (IFP) com a finalidade de testar algoritmos de imageamento em estruturas geológicas complexas. O modelo Marmousi possui fortes variações laterais de velocidade, além disso, apresenta falhas e anomalias de alta velocidade, situações geológicas em que a RTM é indicada. O modelo utilizado possui 560 amostras na direção horizontal e 281 amostras na direção vertical, o espaçamento de malha utilizado foi de $10 \mathrm{~m}$.

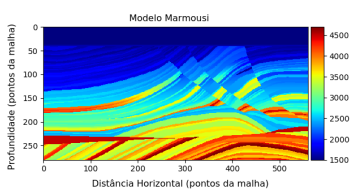

(a)

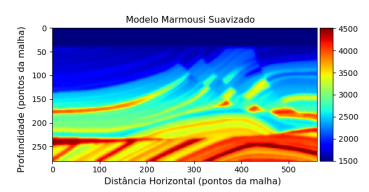

(b)
Figura 4: a) Modelo de velocidade utilizado. b) Modelo de velocidade suavizado.

\section{Dados Sintéticos}

Foram realizados 64 tiros distribuídos na superfície do modelo de velocidade onde receptores foram posicionados. A fonte utilizada foi uma wavelet Ricker com frequência de corte de $30 \mathrm{~Hz}$. As figuras $5 \mathrm{a}$ e $4 \mathrm{~b}$ apresentam dois exemplos de sismogramas sintéticos utilizados no processo de migração utilizando o modelo Marmousi. É importante ressaltar que os dados gerados sinteticamente na modelagem simulam os dados obtidos em uma aquisição sísmica. Esse procedimento é tipicamente utilizado para a validação de algoritmos de imageamento, além disso, evita-se o pré-processamento dos dados para atenuação de ruídos.

\section{Seções Migradas}

O imageamento sísmico é realizado, geralmente, em três etapas: pré condicionamento dos dados, construção do modelo de velocidade e a utilização de uma técnica de migração (ABDELKHALEK, 2013). Nesse experimento,

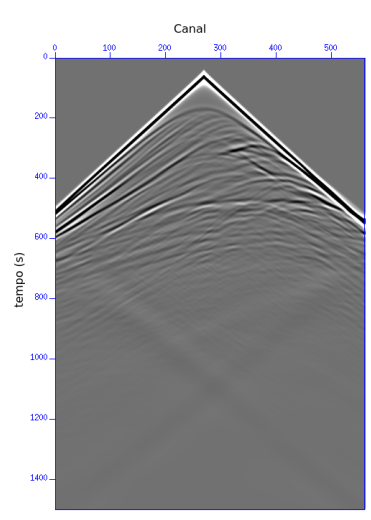

(a)

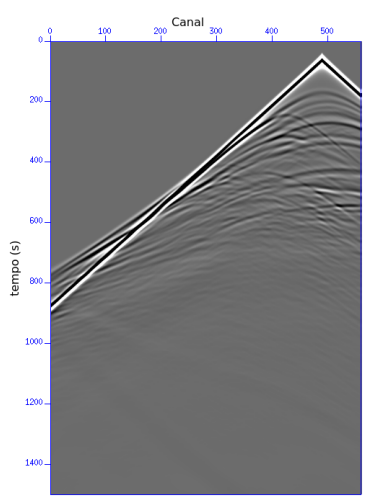

(b)
Figura 5: Sismogramas sintéticos obtidos utilizando o modelo Marmousi.

na etapa de pré condicionamento, as ondas diretas foram silenciadas. O modelo de velocidade utilizado para a construção da matriz de tempo de trânsito e retropropagação dos sismogramas foi o modelo Marmousi suavizado (Figura 5b), pois as grandes variações de velocidade provocam artefatos na matriz de tempo de trânsito, consequentemente, prejudicando a formação da imagem migrada.

A construção da imagem é feita a cada tiro, e empilhadas no final do processo. O número de tiro afeta diretamente a qualidade da imagem. A Figura $6 a$ apresenta o resultado da imagem migrada sem nenhum pós processamento.

\section{Atenuação dos artefatos}

Para atenuação de parte dos artefatos foi limitado o afastamento máximo utilizado em 1000 metros para o empilhamento da imagem final (Figura 6b). Esse processo gera artefatos verticais na seção empilhada devido ao corte abrupto dos longos afastamentos. Entretanto, evitase os artefatos de grandes amplitudes provocados pelos mesmos.

A figura $6 c$ apresenta a seção migrada após a aplicação do filtro passa banda. Os parâmetros do filtro foram selecionados com base na análise do espectro de frequência de forma que as baixas frequências fossem removidas sem prejudicar a imagem das estruturas geológicas.

Por fim foi aplicado o filtro laplaciano na seção migrada após o silenciamento dos longos afastamentos (Figura $6 d$ ). Na utilização do laplaciano foi considerado apenas a direção vertical da derivada para evitar a amplificação dos artefatos provocados pelo silenciamento.

\section{Discussão e conclusão}

A aplicação do filtro laplaciano produziu a melhor imagem migrada, pois remove as baixas frequências enquanto ressalta as frequências mais altas (Figura 7d). Alguns artefatos permanecem na seção migrada, mas são majoritariamente provocados pelos artefatos presentes na matriz de tempo de trânsito. Uma possível solução seria o aumento do número de tiros utilizados na modelagem. 


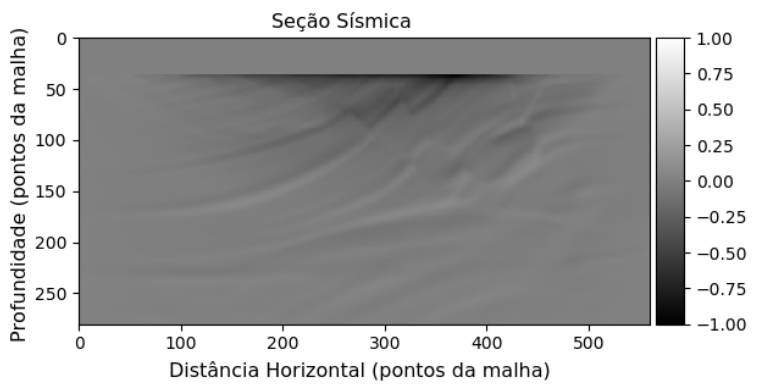

(a)

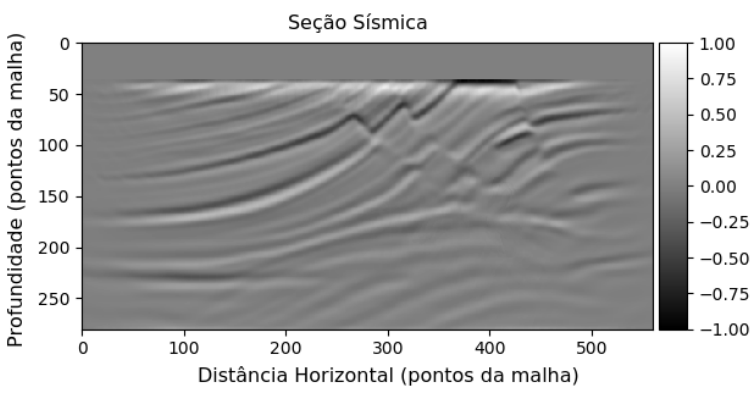

(c)

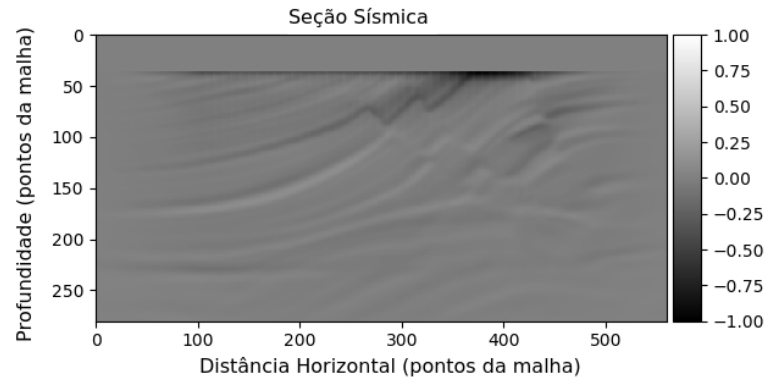

(b)

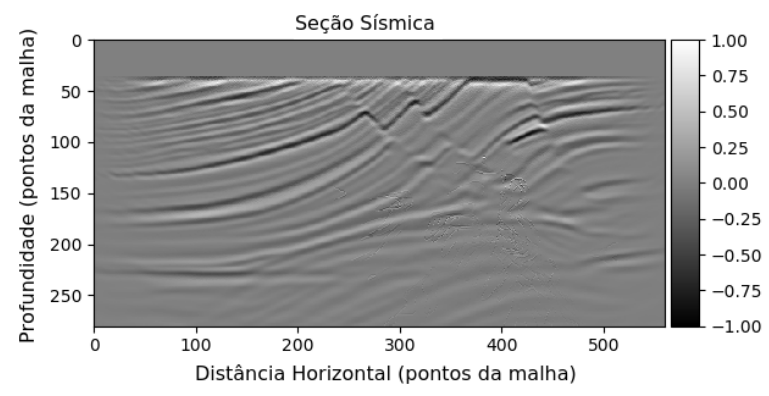

(d)

Figura 6: a) Seções migradas sem nenhum pós processamento. b) Seção migrada com os longos offsets silenciados. c) Seção migrada com os longos offsets silenciados após a aplicação do filtro passa banda. d) Seção migrada com os longos offsets silenciados após a aplicação do filtro laplaciano.

A imagem produzida pelo filtro passa banda apresenta as principais feições geológicas presentes no modelo de velocidade. Entretanto não ressalta todos os refletores que são identificados na seção onde o filtro laplaciano foi aplicado. Essa limitação deve-se à ausências das altas frequências(Figura 7c).

O silenciamento dos longos afastamentos foi fundamental para a atenuação dos ruídos inerentes aos métodos de migração reversa no tempo. Entretanto provocaram artefatos que impediram a aplicação do filtro laplaciano na direção horizontal. Além disso, aplicou-se o silenciamento na região da camada d'água afim de remover os artefatos nas proximidades das fonte.

Esse trabalho foi parte dos estudos iniciais para a implementação e aplicação da RTM. Nos próximos trabalhos serão implementadas diferentes condições de imagens que favoreçam 0 melhor imageamento de estruturas geológicas complexas, como por exemplo a condição por correlação cruzada e suas variantes. Adicionalmente serão utilizados diferentes modelos de velocidade que representem as principais desafios do imageamento sísmico.

\section{Agradecimentos}

Gostaríamos de agradecer o grupo GISIS-UFF, pelo apoio e infraestrutura e a CAPES pelo suporte financeiro.

\section{Referências}

ABDELKHALEK, R. Accélération matérielle pour l'imagerie sismique : modélisation, migration et interprétation. Tese (Doutorado) — L'UNIVERSITÉ DE BORDEAUX I, 2013

BARTOLO, L. di. Modelagem Sísmica Anisotrópica Através do Método das Diferenças Finitas Utilizando Sistemas de Equações em Segunda Ordem. 241 p. Tese (Doutorado) - Universidade Federal do Rio de Janeiro, 2010.

BULCÃO, A. Modelagem e migração reversa 


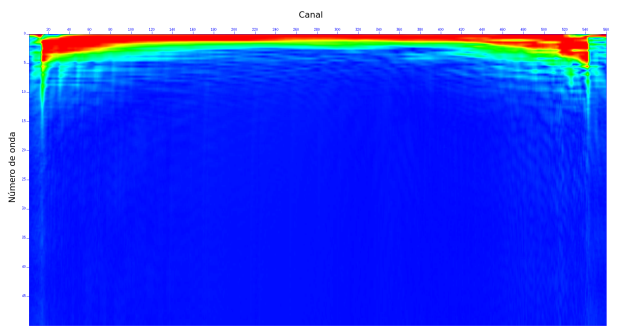

(a)

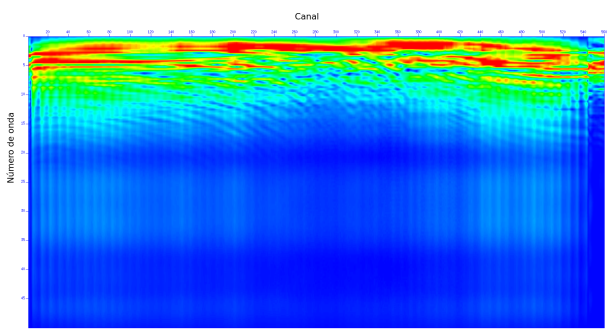

(c)

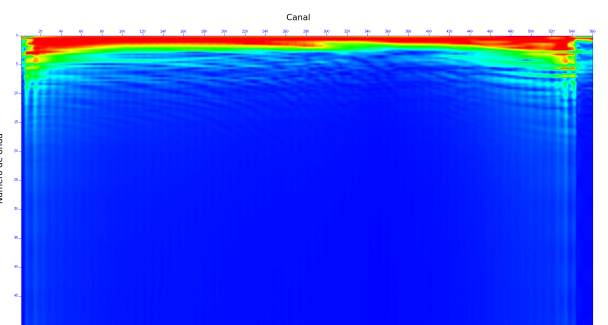

(b)

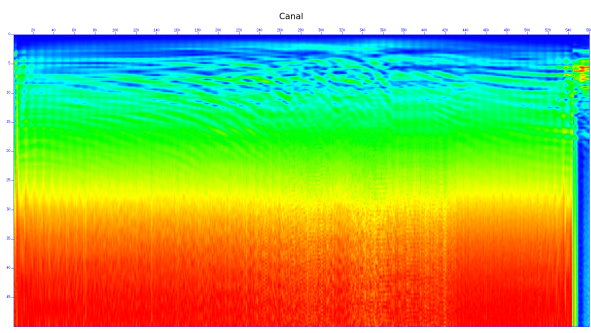

(d)

Figura 7: a) Espectro de frequência da seção migrada sem pós processamento. b) Espectro de frequência da seção migrada com os longos offsets silenciados. c) Espectro de frequência após a aplicação do filtro passa banda. d) Espectro de frequência após a aplicação do filtro laplaciano.

no tempo empregando operadores elásticos e acústicos. Tese (Doutorado) — UFRJ, 2004.

BULCÃO, A.; Soares Filho, D. M.; MANSUR, W. J. Migração Reversa no Tempo 2-D: Estabilidade em relação à Suavização do Modelo de Velocidades e às Reflexões Laterais. 2001.

CLAERBOUT, J. F. Basic Earth Imaging. Book, 2010.

GRAY, S. H. Seismic Imaging. Geophysics, v. 14, p. 15-17, 2001.

GUITTON, A. et al. Least-square Attenuation of Reverse Time Migration Artifacts. In: New Orleans 2006 Annual Meeting SEG. [S.I.: s.n.], 2006. p. 2348-2352.

PANIAGUA, J. G.; QUINTERO, O. L.; SIERRASOSA, D. Laguerre-Gauss Filters in Reverse Time Migration Image Reconstruction. Revista Brasileira de Geofísica, v. 35, n. 2, 2017.

SILVA, M. W. X. Migração reversa no tempo com diferentes condições de imagem. Tese (Doutorado) — UFRJ, 2009.

YILMAZ, Ö. Seismic Data Analysis. [S.I.: s.n.], 2001.

ZHANG, Y.; SUN, J. Practical issues of reverse time migration: true amplitude gathers, noise removal and harmonic- source encoding. In: . [S.I.: s.n.], 2009. 